\title{
PERSPECTIVAS Y EXPERIENCIAS SOBRE EL CONSUMO DE BEBIDAS ALCOHÓLICAS DURANTE EL EMBARAZO: ESTUDIO CUALITATIVO CON EMBARAZADAS DE ESPAÑA, FRANCIA Y PORTUGAL
}

\section{Renata Franco*, Belén Charro**, Maria Raul Xavier}

\section{Resumen}

El trabajo tiene como objetivo explorar las perspectivas y experiencias de las embarazadas españolas, francesas y portuguesas respecto al consumo de alcohol durante la gestación. Se trata de un estudio cualitativo basado en un abordaje fenomenológica. Se realizaron 68 entrevistas en profundidad, procediendo posteriormente a un análisis semiinductivo de los datos con ayuda del programa QSR- NVIVO. Los resultados mostraron semejanzas en los tres países en cuanto a la existencia de consumos de las participantes, así como una información limitada y/o equivocada en cuanto a las consecuencias de la exposición prenatal. Se identificaron también diferencias, sobre todo en cuanto al tipo de bebidas, la presión social o el conocimiento en cuanto a las recomendaciones sobre los consumos. Los datos apuntan la necesidad de abordajes preventivos eficaces que puedan apoyar a las embarazadas en la toma de decisiones informadas.

Palabras-clave: Alcohol, embarazo, perspectivas, conocimientos.

\section{Resumo}

Perspetivas e experiências sobre o consumo de bebidas alcoólicas na gravidez: estudo qualitativo com grávidas de Espanha, França e Portugal

Neste artigo, o objetivo é explorar perspetivas e experiências de grávidas espanholas, francesas e portuguesas quanto ao consumo de álcool durante a gravidez. Trata-se de um estudo qualitativo baseado numa abordagem fenomenológica. Foram realizadas 68 entrevistas em profundidade, procedendo-se posteriormente à análise semi-indutiva dos

* TR3 de CERES - Ethique, Philosophie, Sciences et Société de la Faculté Libre des Lettres et des Sciences humaines, Institut Catholique de Toulouse, France.

Dirección postal: 31, rue de la Fonderie BP 7012 - 31068 Toulouse Cedex 7. França.

Correo Electrónico: fran_re@yahoo.com.br

ORCID: https://orcid.org/0000-0002-7352-2833

** Departamento de Psicología de la Facultad de Ciencias Humanas y Sociales, Universidad Pontificia Comillas (UPC), España.

Dirección postal: Alberto Aguilera, 23 - 28015 Madrid. Espanha.

Correo Electrónico: bcharro@comillas.edu

ORCID: https: //orcid.org/0000-0002-6462-167X

*** Centro de Investigação para o Desenvolvimento Humano (CEDH), Faculdade de Educação e Psicologia, Universidade Católica Portuguesa, Portugal.

Dirección postal: Rua Diogo Botelho, 1327, 4169-005 Porto, Portugal.

Correo Electrónico: mxavier@porto.ucp.pt

ORCID: https://orcid.org/0000-0002-2223-2000 
dados com recurso ao programa QSR-NVIVO. Os resultados mostram semelhanças nos três países quanto à existência de consumos por parte das participantes e informação limitada e/ou errada quanto às consequências da exposição pré-natal. São ainda identificadas diferenças, nomeadamente quanto ao tipo de bebidas, pressão social ou conhecimento quanto a recomendações sobre consumos. Os dados apontam para a necessidade de abordagens preventivas efetivas que possam apoiar a grávida na tomada de decisões informadas.

Palavras-chave: Álcool, gravidez, perspetivas, conhecimentos.

\begin{abstract}
Perspectives and experiences on alcohol consumption during pregnancy: a qualitative study with pregnant women from Spain, France and Portugal

This work aims to explore perspectives and experiences of Spanish, French and Portuguese pregnant women regarding alcohol use during pregnancy. It is a qualitative study based on a phenomenological approach. It included 68 interviews and a semi-inductive data analysis using the QSR-NVIVO software. Results show similarities in the three countries in terms of the participants' consumption and limited and/or wrong information about consequences of prenatal exposure. Differences are also identified, including the type of drinks, social pressure or knowledge regarding recommendations about alcohol use during pregnancy. This work points to the need for effective preventive approaches that can support pregnant women in informed decision-making.
\end{abstract}

Keywords: Alcohol, pregnancy, perspectives, knowledge.

\title{
Introducción
}

En las culturas europeas, el consumo de alcohol se considera un comportamiento habitual, integrado en el estilo de vida y generalmente no considerado como un riesgo para la salud (Gaussot 2004). Los datos de la Organización Mundial de la Salud (OMS o WHO en inglés) muestran que Europa es la región con el consumo de alcohol per cápita más alto del mundo (WHO 2018). Los países europeos varían ampliamente en los patrones de uso. España, Francia y Portugal (países a los que se refiere este documento) y otros países del sur de Europa a menudo se describen con una cultura de consumo diferente a la de los países del norte de Europa. En España, Francia y Portugal, las bebidas alcohólicas están fácilmente disponibles y forman parte de la vida cotidiana de las personas (sobre todo el vino, que a menudo forma parte de las dos comidas diarias principales). A pesar de los cambios recientes (WHO 2019), las tasas de abstinencia son generalmente bajas y la frecuencia de los consumos es mayor, aunque las cantidades consumidas pueden describirse como moderadas (por ej., Anderson y Baumberg 2006).

En España, el alcohol es la primera sustancia psicoactiva consumida y el tabaco la segunda (Observatorio Español de Drogas y Adiciones 2018). También en Francia y Portugal, el alcohol es la sustancia psicoactiva más utilizada (Andler et al. 2018; Balsa, Vital y Urbano 2018). 
En Europa es donde se encuentra el mayor porcentaje de bebedoras de alcohol actuales (WHO 2018), aunque beban con menor frecuencia y cantidad que los hombres. En España, Francia y Portugal también el porcentaje de mujeres que beben es menor que el de los hombres (Andler et al. 2018; Balsa Vital y Urbano 2018; Observatorio Español de Drogas y Adiciones 2018).

Con respecto al uso de alcohol por parte de las mujeres, un tema de particular interés se refiere al consumo durante el embarazo. El alcohol es un agente teratogénico que atraviesa rápidamente la placenta y puede provocar daños en el cerebro y otros órganos del embrión y en el desarrollo del feto (por ej., Popova et al. 2016). La exposición prenatal al alcohol se ha asociado con un conjunto de consecuencias de por vida que en su conjunto son denominadas como Trastornos del Espectro Alcohólico Fetal (TEAF) (por ej., Hoyme et al. 2016). Los datos de una importante revisión sistemática y metaanálisis revelan que 8 de cada 1000 individuos en la población general tienen TEAF (Lange et al. 2017). El TEAF integra problemas neurocognitivos, malformaciones congénitas y otros problemas físicos y de comportamiento, y su manifestación más grave corresponde al diagnóstico de Síndrome Fetal Alcohólico (SAF). El SAF se identificó por primera vez ya en los años 1960 (por ej., Jones y Smith 1973). El consumo de alcohol durante el embarazo es la principal causa de discapacidad mental no genética «evitable» (por ej., Toutain 2010). Popova y sus colegas (Popova et al. 2017) estimaron que la prevalencia de SAF en la población general es de 14,6 por cada 10000 personas y que una de cada 67 mujeres que consumieron alcohol durante el embarazo tendría un hijo con SAF, lo que significa que nacen 119000 niños con SAF en todo el mundo cada año.

Aunque la investigación sobre los efectos asociados con el consumo de alcohol en pequeñas cantidades durante el embarazo no es del todo unánime (por ej., Lundsberg et al. 2015), los estudios apuntan claramente sobre las graves consecuencias en las ingestas de mayores cantidades. Siguiendo un principio de precaución (Conover y Jones 2012), dado que no se conoce una cantidad segura de exposición durante este período, las directrices clínicas de la Organización Mundial de la Salud (WHO 2016) recomiendan no usar bebidas alcohólicas durante el embarazo como la única opción segura (por ej., Míguez, Magri, y Suárez 2009; Elek et al. 2013). Además, estas recomendaciones también promueven la abstinencia de alcohol durante la fase de concepción (Tough et al. 2006; WHO 2016).

Con respecto a las actuaciones de los profesionales que acompañan el embarazo (es decir, médicos/as y enfermeras/as), la OMS también organizó recomendaciones específicas para la identificación y prevención (WHO 2014). Hay estudios (por ej., Kesmodel y Kesmodel 2002) que indican que las mujeres consideran que el enfoque de estos profesionales de la salud ayuda a reducir el consumo. España, Francia y Portugal son tres países en los que existen recomendaciones oficiales que se refieren a la importancia de preguntar a las mujeres embarazadas sobre el consumo y proporcionar información sobre los riesgos asociados (Anglade et al. 
2011; Ministerio de Sanidad, Servicios Sociales e Igualdad 2014; Direção-Geral da Saúde 2015).

A pesar de las recomendaciones, muchas mujeres embarazadas continúan bebiendo alcohol. La prevalencia del consumo de bebidas alcohólicas durante el embarazo en Europa es aproximadamente del 25\%, que es el porcentaje más alto del mundo (Popova et al. 2017).

Los datos epidemiológicos franceses muestran que entre el 0,4\% y el 7,2\% de las mujeres embarazadas hacen algunas transgresiones al estándar «Cero alcohol» para «divertirse» y «no dejar de vivir» (Toutain 2017). Según el Boletín de Salud Pública de Francia (Andler et al. 2018), se estima que uno de cada 1000 nacimientos presenta SAF como consecuencia de la exposición al alcohol durante el embarazo. Hasta donde sabemos, no hay datos representativos a nivel nacional en España. En un estudio realizado en Málaga con 451 mujeres embarazadas, el 27,5\% admitió haber bebido durante el embarazo (Blasco-Alonso et al. 2015). Tampoco en Portugal hay información representativa nacional, aunque en un trabajo realizado por nuestro equipo portugués en este estudio encontró una indicación de consumo durante la gestación del 20,3\% de las mujeres embarazadas $(\mathrm{N}=369)$ en una muestra nacional recolectada en línea (Xavier et al., 2019). Por otro lado, en un estudio del Servicio de Intervenção nos Comportamentos Aditivos e nas Dependências (Carapinha et al. 2015), con una muestra de 1104 mujeres embarazadas de Lisboa y del valle del Tajo, se encontró que el 19,3\% reveló consumir alcohol durante el embarazo.

Las mujeres señalan como algunas de las posibles razones para el consumo de alcohol la poca información y/o información confusa y contradictoria que tienen (por ej., Anderson et al. 2014). En otras ocasiones se trata de problemas previos de alcoholismo, de embarazos no planificado, el estrés, la presión social y grupal y la depresión (por ej., Elek et al. 2013).

Para que la prevención de las consecuencias descritas anteriormente sea cada vez más efectiva, es necesario conocer mejor el fenómeno del consumo de alcohol durante el embarazo, es decir, los factores asociados con estos comportamientos, así como el conocimiento y las perspectivas relacionadas con el consumo de alcohol por las mujeres (Skagerstrøm, Chang y Nilsen 2011). Es en este contexto que se inserta este trabajo, con el objetivo de presentar y discutir las perspectivas y experiencias de embarazadas españolas, francesas y portuguesas con respecto al uso de alcohol durante la gestación. Más específicamente, se pretende explorar las perspectivas y el conocimiento sobre las consecuencias y las pautas sobre el consumo de alcohol durante el embarazo que tienen las mujeres, así como la manera en que abordan esta problemática durante la gestación los profesionales sanitarios. El objetivo es dar a las mujeres una voz con respecto al uso de alcohol en el embarazo desde una mirada intercultural, que es innovadora con respecto a lo que estos tres países (juntos) dicen al respecto. Los objetivos específicos del trabajo conjunto 
realizado por los equipos de investigación de los tres países se describieron previamente en otra publicación (Charro, Xavier y Franco 2019).

\section{Metodología}

Se trata de un estudio con un diseño cualitativo transversal desde un enfoque fenomenológico (Creswell 2007), basado en el significado y experiencia de las participantes en cuanto «peritos experienciales» (Morse 1994) de sus propias vidas. En cuanto diseño cualitativo, pretende la aproximación a cuestiones muy particulares y referidas a una realidad que no puede ser cuantificada por formar parte del universo de los significados, motivos, aspiraciones, creencias y actitudes hacia el fenómeno estudiado (Minayo 2009). Consideramos que el uso de la metodología cualitativa es indispensable para aprehender detalles sobre los factores personales, contextuales y sociales (Ritchie e Lewis 2003) que conducen a las mujeres gestantes a consumir alcohol. El estudio fue aprobado por los Comités de Ética de las Universidades a las que pertenecen las investigadoras.

\section{Participantes}

Las participantes fueron elegidas mediante muestreo no probabilístico por conveniencia, utilizando la metodología de bola de nieve (Ritchie y Lewis 2003; Combessie 2007) mediante el que las participantes indicaban otras gestantes potencialmente interesadas en participar. El tamaño muestral atendió al criterio de saturación teórica (Creswell 2007) y participaron en el estudio 68 mujeres (30 españolas, 20 francesas y 18 portuguesas) que estaban o habían estado embarazadas y recibían seguimiento por parte de los profesionales sanitarios.

En cuanto a las 30 participantes españolas (Madrid), tenían entre 26 y 40 años $(\mathrm{M}=34,26$ y $\mathrm{DP}=3,67)$, casi todas estaban casadas y trabajaban $(90 \%)$ y todas tenían al menos un título universitario (100\%). Para las 20 francesas (Toulouse), la edad mínima era de 24 y la máxima de $45(\mathrm{M}=33,3$ y $\mathrm{DP}=4,6) ; 18$ estaban empleadas (90\%). En cuanto al estado civil, 16 estaban casadas (80\%). La mayoría tenía al menos un título universitario (80\%). Las 18 portuguesas (región norte) tenían entre 20 y 38 años $(M=30,67$ y $\mathrm{DP}=5,21)$. La mayoría estaba casada (94\%) y 12 tenían empleo $(66,5 \%)$, una era estudiante $(5,5 \%)$ y 5 estaban desempleadas $(28 \%)$. La mayoría tenía al menos un título universitario (67\%).

Los criterios de exclusión fueron la dependencia del alcohol u otras sustancias psicoactivas y los embarazos de riesgo, que requieren apoyo exclusivo en un ambiente hospitalario. 


\section{Instrumento}

Se utilizó un guion de entrevista semiestructurada, con preguntas relativas al tema en estudio. El guion fue preparado inicialmente por el equipo portugués (Hoffmeister y Xavier 2018) y luego traducido al español (López-Pinto 2018) y al francés (Fruscione 2017) por los respectivos equipos de los otros dos países participantes. Las tres versiones fueron luego discutidas conjuntamente por las investigadoras responsables de cada país.

El guion también incluía la Prueba de Identificación de Trastornos por Consumo de Alcohol - AUDIT (Saunders et al. 1993) en su versión para entrevista. AUDIT es una herramienta de detección de abuso de alcohol desarrollada por la OMS en colaboración con los Centros de Salud Pública de Australia, Bulgaria, Kenia, México, Noruega y los Estados Unidos (Saunders et al. 1993). En este trabajo, se utilizaron las versiones en español (Rubio et al. 1998), francés (Accietto 2003) y portugués (Cunha 2002). Esta herramienta consta de 10 preguntas. Las preguntas 1 a 3 evalúan el consumo de alcohol (frecuencia y cantidad), las tres siguientes abordan los síntomas de la dependencia y las restantes consecuencias negativas del consumo. Cada pregunta varía de 0 a 4 puntos y el total máximo es de 40 puntos. AUDIT se diseñó inicialmente para su uso en la Atención Primaria de Salud y se ha estudiado en una variedad de entornos para detectar diferentes niveles de consumo de alcohol de forma rápida y sencilla. En este estudio, AUDIT se adaptó al objetivo propuesto: cada una de las preguntas se subdividió en dos, la primera referida al período de vida antes del embarazo y la segunda al período actual del embarazo, lo que permitió explorar las posibles diferencias.

La guía de entrevistas, además de las preguntas de carácter sociodemográfico, consistió en preguntas relacionadas con los siguientes temas: el consumo de alcohol y sus circunstancias; información y conocimientos sobre las consecuencias del consumo de alcohol durante el embarazo; evaluación y prevención selectiva (incluida la información disponible en el contexto de la atención sanitaria); indicadas y universales.

\section{Procedimiento}

Las 68 participantes recibieron explicaciones sobre el estudio, sobre todo sobre su objetivo, el tratamiento de los datos, la naturaleza voluntaria de la participación, el anonimato y la confidencialidad. Todas firmaron un Formulario de Consentimiento Informado que incluía la autorización para grabar las entrevistas. Estas se realizaron de forma individual, en cada uno de los países siempre por el mismo investigador, y duraron un promedio de 45 minutos.

Posteriormente, se transcribieron íntegramente las entrevistas y se analizó su contenido (análisis categórico e interpretativo) según un enfoque semiinductivo, 
utilizando el programa QSR-NVIVO v.11. En cada país, a partir del discurso de las participantes, se organizó una primera versión de las categorías inspiradas en la Teoría Fundamentada (Strauss y Corbin 1994). En un procedimiento de triangulación (Ruiz Olabuénaga 2008), una de las investigadoras, leyó todas las entrevistas e hizo una primera identificación de los códigos y categorías de análisis. A medida que el análisis avanzaba, la primera codificación se reformuló en base a las relaciones entre las categorías que surgieron del discurso de las participantes. Otra(s) investigador(as) de cada país revisó(aron) el sistema categórico para comparar el material recopilado hasta que se llegó a un acuerdo sobre la codificación de todas las entrevistas. Las tres investigadoras principales de cada país también discutieron los tres sistemas de categorías. Los procedimientos de análisis de datos revelan el uso de la triangulación del investigador (p. ex., Patton 1999).

El conjunto resultante de categorías y subcategorías analíticas se organizaron en torno a ocho dimensiones o nodos temáticos: (1) consumo pregestacional; (2) consumo durante el embarazo; (3) autodeclaración (abstinencia; facilidad/dificultad para hablar sobre el consumo; razón del consumo; contexto en el que ocurre el consumo; lo que consideran «consumo aceptable»; opiniones sobre el consumo de otras mujeres embarazadas); (4) información sobre los efectos del consumo durante el embarazo y fuente(s) de información; (5) consumo en el contexto familiar y social; (6) monitoreo de los profesionales de la salud; (7) prevención universal; (8) prevención selectiva e indicada.

Las dimensiones y sus subcategorías relacionadas con los objetivos de este trabajo serán presentadas y discutidas a continuación.

\section{Resultados y discusión}

El análisis de los resultados del AUDIT para los consumos descritos antes del embarazo y durante el embarazo reflejó un cambio en el consumo para todas las mujeres que describieron beber alcohol antes del embarazo. En España, el 66\% redujo el consumo, mientras que el 34\% lo abandonó por completo; en Francia y Portugal, las tasas de retirada total fueron más altas: 70\% y 75\% respectivamente. Estos datos parecen demostrar que el embarazo tiende a actuar como factor de protección contra el consumo de alcohol, lo cual ya había sido señalado por un estudio realizado en Portugal por el Serviço de Intervenção nos Comportamentos Aditivos e nas Dependências (Carapinha et al. 2015).

Otro aspecto identificado en los tres países, y asociado a la abstinencia de la mujer embarazada, es el apoyo del marido/pareja. La mayoría de los participantes que no beben alcohol durante el embarazo reciben apoyo de su pareja (implícita o explícitamente). En este sentido, Van der Wulp, Hoving y Vries (2015) consideraron la relación de pareja como un factor importante en la ausencia de consumo de alcohol. 
Aunque la reducción y /o abstinencia se evidenció en el discurso de las participantes, durante las entrevistas se identificaron varios matices sobre el consumo de alcohol durante el embarazo. Analizando los consumos descritos, se trata de dosis reducidas y situaciones esporádicas, en un patrón que podríamos describir como consumo social. Si esto no ocurriera durante la gestación no se consideraría un problema. Sin embargo, al ocurrir durante el embarazo, estos datos revelan que no se están cumpliendo las indicaciones nacionales e internacionales para no consumir alcohol durante el embarazo (WHO 2016).

Las participantes de los tres países que se permitieron desviarse de las indicaciones de que no se debe consumir alcohol durante el embarazo presentaron a veces en sus discursos la falsa creencia de que el consumo ocasional y en bajas dosis no afectaría a la salud del feto. Los resultados coinciden con el trabajo de Hammer y Inglin (2014) en el que se demostró que este tipo de falsa creencia aumenta en ausencia de información correcta y actualizada sobre el riesgo asociado al consumo durante el embarazo. En nuestro estudio, cuando se interrogó a las mujeres sobre las consecuencias del consumo de alcohol durante el embarazo, la mayoría de ellas mostraron poca información sobre el tema y un conocimiento vago o incluso erróneo. Algunas participantes consideraron que la regla de abstinencia se aplicaba sólo en los tres primeros meses de embarazo, otras dijeron que el consumo de alcohol era menos perjudicial al final del embarazo, otras afirmaron que el consumo de alcohol durante los tres primeros meses no era grave presentando justificaciones no científicas y otras asumieron claramente la falta de información. Estos datos están en línea con los indicados en un estudio anterior (Toutain 2010). La desvalorización de los consumos (particularmente en los casos de dosis más bajas) identificada en el discurso de algunas participantes de los tres países puede estar vinculada a su concepto de abstinencia, como lo afirma Toutain (2010), que describe que el concepto de abstinencia a menudo no se percibe como abstinencia total.

En los tres países, participantes informaron de que los amigos, los miembros de la familia y la pareja/marido (a menudo también identificados por las propias mujeres como un factor de protección contra el consumo de alcohol), son los que más fomentan o apoyan el consumo ocasional en entornos festivos por parte de las mujeres embarazadas. Dupraz et al. (2013) ya habían señalado que la invitación a beber alcohol hecha por un pariente cercano está fuertemente asociada con el consumo de alcohol en el embarazo.

Se puede observar que, en general, la abstinencia tiende a ser una regla en la vida cotidiana de las mujeres, pero en un contexto festivo la transgresión es ejercida y compartida por varios actores que participan en la vida de la mujer embarazada. En los tres países, se identificó la presión social para beber, y en Portugal todas las mujeres informaron que la sentían. Se encontraron resultados similares en el trabajo de Meurk et al. (2014). 
La normalización cultural del consumo puede estar relacionada con el desconocimiento o la falta de importancia que se da a las indicaciones de no beber alcohol durante el embarazo. Skagerstrøm, Chang y Nilsen (2011) afirman en su revisión sistemática de 14 estudios internacionales que las normas culturales sobre el consumo de alcohol tienden a tener más influencia en el consumo que las restricciones legales. Esta noción es importante para mejorar el tipo de intervención preventiva en este campo.

Otra similitud entre España, Francia y Portugal se refiere a la falta de información recibida en las consultas de seguimiento durante el embarazo. Hammer y Inglin (2014) también encontraron datos similares, e incluso describieron que estos profesionales llegaban a indicar el consumo esporádico como apropiado. Las participantes de España y Francia destacaron la permisividad de las recomendaciones recibidas por algunos profesionales de la salud. En Portugal, los médicos (cuando abordan el tema) parecen ser más restrictivos en comparación con las enfermeras. Los resultados coinciden, por ejemplo, con los datos obtenidos por Hammer y Inglin (2014), donde la mayoría de las encuestadas dicen que no recibieron información proporcionada por los profesionales de la salud sobre los riesgos del consumo de alcohol. De manera similar, el estudio de Dupraz et al. (2013) reveló que menos de la mitad de las participantes tenían a su médico de cabecera o ginecólogo como fuente de información. En Portugal, ninguna de las participantes parece conocer alguna guía para las mujeres embarazadas hacia la abstinencia total. Los datos apuntan al incumplimiento por parte de varios profesionales de las indicaciones existentes sobre cómo deben actuar en los momentos de contacto/consulta con las mujeres gestantes (en los tres países) y las recomendaciones para evitar el consumo durante el embarazo no llegan a un gran porcentaje de las mujeres embarazadas, como ya describieron Mendoza et al. (2019). A la vista de los datos obtenidos, el principio de precaución (Department of Health 2016) presente en las recomendaciones difundidas (WHO 2016) no parece inspirar las opciones de muchas mujeres gestantes en los tres países estudiados.

Se encontraron diferencias entre los tres países en cuanto a las bebidas alcohólicas más consumidas por las mujeres embarazadas: en España, la bebida más mencionada fue la cerveza, mientras que en Francia y Portugal las participantes indicaron el vino. Estos datos coinciden con los de April et al. (2010), quienes observaron que el vino, el champán y la cerveza se consideraban menos perjudiciales durante el embarazo que los «alcoholes fuertes». En cuanto a la prevención, las participantes francesas tendieron a valorar la información preventiva, como por ejemplo la advertencia gráfica contra el alcohol durante el embarazo presente en las botellas de vino. En España, las participantes consideraron que las campañas publicitarias eran más útiles como medio de difusión de la información sobre el tema, ya que eran más accesibles y tenían un mayor impacto social. También en Portugal las participantes describieron como más útiles los anuncios que aparecen señalados en las botellas. 
En general, los resultados de este estudio permitieron identificar similitudes en los discursos de las participantes de los tres países, pero los resultados también revelan fenómenos individuales más complejos vinculados a vulnerabilidades psicológicas que pueden favorecer el consumo de alcohol durante el embarazo. Por ejemplo, la ansiedad ante el embarazo y los cambios relacionados con la maternidad fue citada por las entrevistadas francesas como otra razón para beber. Estos datos se observaron sólo en mujeres francesas que estaban embarazadas por segunda vez, lo que mostró una sobrecarga asociada a las tareas relacionadas con el cuidado del primer hijo. Skagerstrøm, Chang y Nilsen (2011) también destacan la exposición a situaciones de alta ansiedad como predictor del consumo de alcohol durante el embarazo.

Por lo tanto, es necesario seguir investigando el fenómeno del consumo de alcohol durante el embarazo para poder lograr una comprensión más completa de los factores asociados con el consumo durante este período de la vida.

\section{Conclusiones}

Varios estudios advierten del problema del alcoholismo durante el embarazo (por ej., Beck et al. 2013; Dumas, Lejeune y Simmat-Durand 2014), pero pocos abordan específicamente el tema de la bebida moderada y recreativa. Así, este trabajo representa una contribución al tema, como pionero en la discusión conjunta de tres países del sur de Europa. En cuanto a la metodología utilizada, destacamos como puntos fuertes la triangulación (Patton 1999) de fuentes (de tres países) e investigadores, así como el logro de la saturación teórica (Creswell 2007).

En cuanto a los resultados obtenidos, destacamos que las perspectivas y experiencias sobre el consumo de alcohol durante el embarazo presentan puntos comunes y diferencias en las intervenciones de las participantes de España, Francia y Portugal. Los resultados mostraron fallas y/o falta de información recibida durante las consultas de seguimiento del embarazo en los contactos con médicos/as y enfermeros/as. Las creencias falsas, la información errónea o inexistente, así como la presión social asociada a sus parejas y al entorno en el que viven las mujeres gestantes también pueden ser obstáculos para la abstinencia. En los tres países, el tipo y la calidad de la información no parece permitir una decisión claramente informada sobre el uso del alcohol durante el embarazo. Aunque muchas participantes que reportaron beber antes del embarazo habían dejado de hacerlo, otras señalaron «beber socialmente» durante el embarazo. Esto no está de acuerdo con las directrices de la OMS (WHO 2016) y confirma que hay mujeres que beben durante la gestación, como revelan los datos presentados, por ejemplo, por Popova et al. (2017).

Para asegurar que todas las mujeres (embarazadas) conozcan las recomendaciones existentes en cuanto al consumo de alcohol durante la gestación y exista una 
decisión informada, destacamos la importancia de que este fenómeno siga siendo estudiado. Los datos resultantes de la investigación científica son de suma importancia para la organización de estrategias de prevención cada vez más eficaces.

\section{Referencias bibliográficas}

Accietto, Cataldo. 2003. «La validation d'une version française du questionnaire A.U.D.I.T. «Alcohol Use Identification Test»». Tesis de doctorado. Université de Genève. DOI: https://doi.org/10.13097/archive-ouverte/unige:174

Anderson, Peter, y Ben Baumberg. 2006. Alcohol in Europe. London: Institute of Alcohol Studies.

Anderson, Amy, Alexis Hure, Frances Kay-Lambkin, y Deborah Loxton. 2014. «Women's perceptions of information about alcohol use during pregnancy: a qualitative study». BMC Public Health 14: 10-48. DOI: https://doi.org/10.1186/1471-2458-14-1048

Andler, Raphaël, Chloé Cogordan, Jean-Baptiste Richard, Virginie Demiguel, Nolwenn Regnault, Romain Guignard, Anne Pasquereau, y Viêt Nguyen-Thanh. 2018. Baromètre santé 2017: Consommations d'alcool et de tabac durant la grossesse. Saint-Maurice: Santé publique France.

Anglade, Cécile, Françoise Bicheron, Juliette Bloch, Véronique Brossard, David Campgil, y Francine Caumel-Dauphin. 2011. Alcool et grossesse, parlons-en: guide à l'usage des professionnels. Paris: Ministère du Travail, de l'Emploi et de la Santé.

April, Nicole, Chantale Audet, Louise Guyon, y Hélène Gagnon. 2010. «Représentations sociales et consommation d'alcool pendant la grossesse». Drogues, Santé et Société 9 (2): 17-48. DOI: https://doi.org/10.7202/1005299ar

Balsa, Casimiro, Clara Vital, y Claudia Urbano. 2018. IV Inquérito Nacional ao Consumo de Substâncias Psicoativas na População Geral, Portugal 2016/17. Lisboa: SICAD - Serviço de Intervenção nos Comportamentos Aditivos e nas Dependências. Disponível em http: //www.sicad.pt/BK/EstatisticaInvestigacao/EstudosConcluidos/Lists / SICAD_ESTUDOS/Attachments/181/IV INPG 2016_17_PT.pdf

Blasco-Alonso, Marta, Ernesto González-Mesa, Milagros Gálvez Montes, Isabel Lozano Bravo, Federico Merino Galdón, Francisco Cuenca Campos et al. 2015. «Exposición a tabaco, alcohol y drogas de abuso en gestantes: Estudio de prevalencia en gestantes de Málaga (España)». Adicciones 1 (27): 99-108. DOI: https://doi.org/10.20882/adicciones.695

Beck, François, Jean-Baptiste Richard, Agnès Dumas, Laurence Simmat-Durand, y Stéphanie Vandentorren. 2013. «Enquête sur la consommation de substances psychoactives des femmes enceintes». La Santé en Action 423, 5-7. Disponible en https://www.santepubliquefrance.fr/docs/la-sante-en-action-jan-mars-2013-n-423-sante-sexuelle-a-quelsprofessionnels-s-adresser

Carapinha, Ludmila, Carla Ribeiro, Elsa Lavado, Mário Castro, y Cristina Ribeiro. 2015. O Consumo de álcool na Gravidez. Lisboa: SICAD - Serviço de Intervenção nos Comportamentos Aditivos e nas Dependências. Disponible en http://www.sicad.pt/PT/EstatisticaInvestigacao /EstudosConcluidos $/$ Paginas /detalhe.aspx?itemId=157\&lista=SICAD_ESTUDOS\&bkUrl=/BK/EstatisticaInvestigacao/EstudosConcluido

Charro, Belén, Maria Raúl Xavier, y Renata Franco. 2019. «Embarazo y alcohol: estudio cualitativo sobre los conocimientos y actitudes de las mujeres gestantes en España, Francia y Portugal». In Atas do $8 .^{\circ}$ Congresso Ibero-Americano em Investigação 
Qualitativa - Investigação Qualitativa na Saúde (CIAIQ2019), vol. 2., organizado por A. P. Costa, C. Oliveira, E. Synthia, J. Ribeiro, H. Presado, C. Baixinho, 669-674. Lisboa: Ludomedia. Disponible en https://proceedings.ciaiq.org/index.php/CIAIQ2019/ article/view/2138

Combessie, Jean-Claude. 2007. La méthode en sociologie. 5. ${ }^{a}$ ed. Paris: La Découverte.

Conover, Elisabeth, y Kenneth Jones. 2012. «Safety concerns regarding binge drinking in pregnancy: A review: Birth Defects Birth Research Part A». Clinical and Molecular Teratology 94: 570-575. DOI: https://doi.org/10.1002/bdra.23034

Creswell, John. 2007. Qualitative inquiry and research design: Choosing among five traditions. Thousand Oaks, CA: Sage.

Cunha, João. 2002. Validação da versão portuguesa dos Questionários AUDIT e Five-Shot para identificação de consumo excessivo de álcool. Lisboa: Internato Complementar de Clínica Geral da Zona Sul.

Department of Health 2016. «Alcohol guidelines review-Report from the Guidelines Development Group to the UK Chief Medical Officers». Disponible en https://assets. publishing.service.gov.uk/government/uploads/system/uploads/attachment_data/ file/545739/GDG_report-Jan2016.pdf [Consultado en 13 de noviembre de 2019].

Direção-Geral da Saúde. 2015. Programa Nacional para a Vigilância da Gravidez de Baixo Risco. Lisboa: Direção-Geral da Saúde.

Dumas, Agnès, Claude Lejeune, y Laurence Simmat-Durand. 2014. «Tabac, alcool et cannabis pendant la grossesse: qui sont les femmes à risque?» Santé Publique 26: 603-612. DOI : https://doi.org/10.3917/spub.145.0603

Dupraz, Julien, Valérie Graff, Judith Barasche, Jean-François Etter, y Michel Boulvain. 2013. «Tobacco and alcohol during pregnancy: prevalence and determinants in Geneva 2008». Swiss Medical Weekly 143: 1-8. DOI: https://doi.org/10.4414/smw.2013.13795

Elek, Elvira, Shelly Harris, Claudia Squire, Marjorie Margolis, Mary Kate Weber, Elizabeth Parra Dang, y Betsy Mitchell. 2013. «Women's knowledge, views, and experiences regarding alcohol use and pregnancy: opportunities to improve health messages». American Journal of Health Education 44(4): 177-190. DOI: https://doi.org/10.1080/1932 5037.2013.768906

Fruscione, Margaux. (2017). «Attitude vis-à-vis de la consommation d'alcool durant la grossesse: étude interculturelle menée en France». Tesis de maestría en psicología. Institut Catholique de Toulouse.

Gaussot, Ludovic. 2004. Modération et sobriété: Etudes sur les usages sociaux de l'alcool. Paris: L'Harmattan.

Hammer, Rapaël, y Inglin Sophie. 2014. «'I don't think it's risky, but...' pregnant women's risk perceptions of maternal drinking and smoking». Health, Risk E Society 16 (1): 22-35. DOI: https://doi.org/10.1080/13698575.2013.863851

Hoffmeister, Kristen, y Maria Raúl Xavier. 2018. «Consumo de álcool durante a gravidez Perceção de mulheres grávidas relativamente à informação disponível (Estudo exploratório qualitativo)». In Actas do 3. ${ }^{\circ}$ Congresso da Ordem dos Psicólogos Portugueses, 637647. Lisboa: Ordem dos Psicólogos Portugueses. Disponible en http://hdl.handle. net $/ 10400.14 / 25683$

Hoyme, Eugene, Wendy Kalberg, Amy Elliott, Jason Blankenship, David Buckley, AnnaSusan Marais, Melanie Manning et al. 2016. «Updated Clinical Guidelines for Diagnosing Fetal Alcohol Spectrum Disorders». Pediatrics 138 (2): 1-18. DOI: https://doi. org/10.1542/peds.2015-4256 
Jones, Kenneth L., y David W. Smith. 1973. «Recognition of the fetal alcohol syndrome in early infancy». Lancet 302 (7836): 999-1001. DOI: https://doi.org/10.1016/S01406736(73)91092-1

Kesmodel, Ulrik, y Schiøler Pia Kesmodel. 2002. «Drinking during pregnancy: attitudes and knowledge among pregnant Danish women, 1998». Alcohol Clinical and Experimental Research 26 (10): 1553-1560. DOI: https://doi.org/10.1111/j.1530-0277.2002.tb02455.x

Lange, Shannon, Charlotte Probst, Gerrit Gmel, Jürgen Rehm, Larry Burd, y Svetlana Popova. 2017. «Global Prevalence of Fetal Alcohol Spectrum Disorder Among Children and Youth A Systematic Review and Meta-analysis». JAMA Pediatrics 171(10): 948-956. DOI: https://doi.org/10.1001/jamapediatrics.2017.1919

López-Pinto, Isabel. 2018. «Consumo de alcohol durante el embarazo: la perspectiva de las mujeres». Trabajo de Fin de Máster en Psicología General Sanitaria. Universidad Pontificia Comillas. Madrid.

Lundsberg, Lisbet, Jessica L. Illuzzi, Kathleen Belanger, Elizabeth Triche, y Michael Bracken. 2015. «Low-to-moderate prenatal alcohol consumption and the risk of selected birth outcomes: A prospective cohort study». Annals of Epidemiology 25 (1): 46-54.e3. DOI: https://doi.org/10.1016/j.annepidem.2014.10.011

Mendoza, Ramón, Elena Morales Marente, Soledad Palacios, Carmen Rodríguez-Reinado, Isabel Corrales-Gutiérrez, y Óscar García-Algar. 2019. «Health advice on alcohol consumption in pregnant women in Seville (Spain)». Gaceta Sanitaria 1 (33): 1-10. DOI: https://doi.org/10.1016/j.gaceta.2018.11.008

Meurk, Carla, Alex Broom, Jon Adams, Wayne Hall, y Jayne Lucke. 2014. «Factors influencing women's decisions to drink alcohol during pregnancy: Findings of a qualitative study with implications for health communication». BMC Pregnancy and Childbirth 14 (1): 2-9. DOI: https: //doi.org/10.1186/1471-2393-14-246

Míguez, Hugo, Raquel Magri, y Mónica Suárez. 2009. «Consumo de tabaco y bebidas alcohólicas durante el embarazo». Acta Psiquiátrica y Psicológica de América Latina 55(2): 76-83.

Minayo, María C. de S. 2009. Pesquisa Social: Teoria, método e criatividade. Petrópolis: Vozes.

Ministerio de Sanidad, Servicios Sociales e Igualdad. 2014. Guía de práctica clínica de atención en el embarazo y puerperio. Andalucía: Agencia de Evaluación de Tecnologías Sanitarias de Andalucía (AETSA). Disponible en https://portal.guiasalud.es/gpc/embarazo-puerperio

Morse, Janice. 1994. Designing Funded Qualitative Research. London: Sage.

Observatorio Español de las Drogas y Adicciones. 2018. XII Encuesta sobre Alcohol y otras Drogas en España (EDADES) 2017-2018. Madrid: Ministerio de Sanidad, Consumo y Bienestar Social. Disponible en https://pnsd.sanidad.gob.es/profesionales/sistemasInformacion/sistemaInformacion/pdf/EDADES_2017_Informe.pdf

Patton, Michale Quin. 1999. «Enhancing the quality and credibility of qualitative analysis». Health Services Research 34 (5 Part 2): 1189-1208. Disponible en https://www.ncbi.nlm. nih.gov/pmc/articles/PMC1089059

Popova, Svletana, Shannon Lange, Larry Burd, y Jürgen Rehm. 2016. «The Economic Burden of Fetal Alcohol Spectrum Disorder in Canada in 2013». Alcohol and Alcoholism 51 (3): 367-375. DOI: https://doi.org/10.1093/alcalc/agv117

Popova, Svetlana, Shannon Lange, Charlotte Probst, Gerrit Gmel, y Jürgen Rehm. 2017. «Estimation of national, regional, and global prevalence of alcohol use during pregnancy and fetal alcohol syndrome: a systematic review and meta-analysis». Lancet Glob Health 5(3): 290-299. DOI: https://doi.org/10.1016/S2214-109X(17)30021-9 
Ritchie, Jane, y Jane Lewis. 2003. Qualitative research practice: A guide for social science students and researchers. London: Sage.

Rubio Valladolid, Vicedo Bermejo, Sanchez Serrano Caballero, y Carrasco Santo Domingo. 1998. «Validación de la prueba para la identificación de trastornos por uso de alcohol (AUDIT) en Atención Primaria». Revista Clínica Española 198: 11-14.

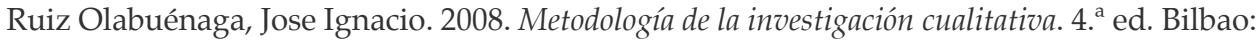
Ediciones de la Universidad de Deusto.

Saunders, John, Olaf G. Aasland, Thomas F. Babor, Juan R. de la Fuente, Marcus Grant. 1993. «Development of the alcohol use disorders identification test (AUDIT): WHO Collaborative Project on Early Detection of Persons with Harmful Alcohol Consumption-II». Addiction 88 (6): 791-804. DOI: https://doi.org/10.1111/j.1360-0443.1993.tb02093.x

Skagerstrøm, Jana, Grace Chang, y Per Nilsen. 2011. «Predictors of drinking during pregnancy: a systematic review». Journal of Women's Health 20(6): 901-913. DOI: https://doi. org/10.1089/jwh.2010.2216

Strauss, Anselm, y Juliet Corbin. 1994. «Grounded theory methodology: An overview». In Handbook of qualitative research, editado por Norman K. Denzin y Yvona S. Lincoln, 273-285. Thousand Oaks, CA: Sage.

Tough, Suzanne, Karen Tofflemire, Margaret Clarke, y Christine Newburn-Cook. 2006. «Do women change their drinking behaviors while trying to conceive? An opportunity for preconception counseling». Clinical Medicine and Research 4(2): 97-105. DOI: https: // doi.org/10.3121/cmr.4.2.97

Toutain, Stéphanie. 2010. «What women in France say about alcohol abstinence during pregnancy»? Drug and Alcohol Review 29: 184-188.

Toutain, Stéphanie. 2017. «Concilier 'Zéro alcool pendant la grossesse' et alcoolisation ponctuelle importante des premières semaines. Une enquête qualitative sur des forums de discussion». Bulletin Epidémiologique Hebdomadaire 11: 207-212. Disponible en http:// beh.santepubliquefrance.fr/beh/2017/11/2017_11_2.html

van der Wulp, Nickie, Cisca Hoving, y Hein de Vries. 2015. «Partner's Influences and Other Correlates of Prenatal Alcohol Use». Maternal and Child Health Journal 19: 908-916. DOI: https://doi.org/10.1007/s10995-014-1592-y

WHO - World Health Organization. 2014. Guidelines for the identification and management of substance use and substance use disorders in pregnancy. Genève: WHO. Disponible en https://apps.who.int/iris/handle/10665/107130

WHO - World Health Organization. 2016. Prevention of harm caused by alcohol exposure in pregnancy. Rapid review and case studies from Member States. Copenhague: WHO Regional Office for Europe. Disponible en https://apps.who.int/iris/handle/10665/ 329491

WHO - World Health Organization. 2018. Global status report on alcohol and health 2018. Genève: WHO. Disponible en https://apps.who.int/iris/handle/10665/274603

WHO - World Health Organization. 2019. Status report on alcohol consumption, harm and policy responses in 30 European countries 2019. Copenhagen: WHO Regional Office for Europe. Disponible en http://www.euro.who.int/en/health-topics/disease-prevention/alcohol-use/publications/2019/status-report-on-alcohol-consumption,-harm-and-policy-responses-in-30-european-countries-2019

Xavier, Maria Raúl, Bárbaro Claro, Carla Martins, y Pedro Dias. 2019. «Alcohol use during pregnancy - a Portuguese study».8th International Conference on Fetal Alcohol Spectrum Disorder Syllabus Book, 34. Vancouver, The University of British Columbia, 6-9 de marzo de 2019. Disponible en https://www.fasportalen.se/Portals/0/FASD2019_Syllabus.pdf 
Renata Franco. Doctora en Evaluación Psicológica por la Universidad São Francisco (USF). Es profesora del postgrado en Psicología Clínica del Institut Catholique de Toulouse (Francia) e investigadora del Laboratorio Ethique, Philosophie, Sciences et Société (TR3) de esa misma universidad. Participa en el área académica, investigando e impartiendo cursos dentro del área de la Psicología Clínica, la Técnicas Proyectivas, Evaluación de la personalidad y Drogodependencias.

Endereço Eletrónico: fran_re@yahoo.com.br

Belén Charro. Doctora en Psicología, profesora Propia Agregada en la Facultad de Ciencias Humanas y Sociales de la Universidad Pontificia Comillas de las asignaturas Evaluación Psicológica y Psicología de las Adicciones. Es Directora del Máster en Psicología General Sanitaria de esa universidad. Ha participado y dirigido diversos proyectos de investigación relacionados con el consumo de drogas y alcohol.

Endereço Eletrónico: bcharro@comillas.edu

Maria Raul Xavier. Profesora Auxiliar de la Faculdade de Educação e Psicologia, Universidade Católica Portuguesa (FEP-UCP). Licenciada en Psicología y doctora en Ciencias Biomédicas pela Univ. do Porto. Es investigadora en el Centre for Studies in Human Development (CEDH), Universidade Católica Portuguesa. Su campo de investigación se centra en el embarazo, maternidad y primeros años de los niños, así como en el consumo de alcohol.

Endereço Eletrónico: mxavier@porto.ucp.pt

Artículo recibido el 2 de febrero y aceptado para su publicación el 4 de junio de 2020. 